\title{
Video Article \\ Breathing-controlled Electrical Stimulation (BreEStim) for Management of Neuropathic Pain and Spasticity
}

\author{
Sheng $\mathrm{Li}^{1,2,3}$ \\ ${ }^{1}$ Department of Physical Medicine and Rehabilitation, University of Texas Health Science Center at Houston \\ ${ }^{2}$ UTHealth Motor Recovery Laboratory, TIRR Memorial Hermann Hospital \\ ${ }^{3}$ The Institute of Rehabilitation and Research (TIRR), TIRR Memorial Hermann Hospital
}

Correspondence to: Sheng Li at sheng.li@uth.tmc.edu

URL: https://www.jove.com/video/50077

DOI: doi: $10.3791 / 50077$

Keywords: Medicine, Issue 71, Neuroscience, Neurobiology, Anatomy, Physiology, Behavior, electrical stimulation, BreEStim, electrode, voluntary breathing, respiration, inspiration, pain, neuropathic pain, pain management, spasticity, stroke, spinal cord injury, brain, central nervous system, CNS, clinical, electromyogram, neuromuscular electrical stimulation

Date Published: 1/10/2013

Citation: Li, S. Breathing-controlled Electrical Stimulation (BreEStim) for Management of Neuropathic Pain and Spasticity. J. Vis. Exp. (71), e50077, doi:10.3791/50077 (2013).

\section{Abstract}

Electrical stimulation (EStim) refers to the application of electrical current to muscles or nerves in order to achieve functional and therapeutic goals. It has been extensively used in various clinical settings. Based upon recent discoveries related to the systemic effects of voluntary breathing and intrinsic physiological interactions among systems during voluntary breathing, a new EStim protocol, Breathing-controlled Electrical Stimulation (BreEStim), has been developed to augment the effects of electrical stimulation. In BreEStim, a single-pulse electrical stimulus is triggered and delivered to the target area when the airflow rate of an isolated voluntary inspiration reaches the threshold. BreEStim integrates intrinsic physiological interactions that are activated during voluntary breathing and has demonstrated excellent clinical efficacy. Two representative applications of BreEStim are reported with detailed protocols: management of post-stroke finger flexor spasticity and neuropathic pain in spinal cord injury.

\section{Video Link}

The video component of this article can be found at https://www.jove.com/video/50077/

\section{Introduction}

Electrical stimulation (EStim) refers to the application of electrical current to muscles or nerves in order to achieve functional and therapeutic goals. It has been extensively used in various clinical settings, e.g., transcutaneous electrical nerve stimulation (TENS) for pain management ${ }^{1}$, peroneal nerve stimulation for foot drop ${ }^{2}$, neuromuscular electrical stimulation (NMES) for activation and strengthening of paralyzed or weakened muscles ${ }^{3}$. When NMES is used to achieve a functional task, it is termed functional electrical stimulation (FES) ${ }^{4}$. Electromyogram (EMG)-triggered neuromuscular stimulation has been used to augment effectiveness of electrical stimulation in motor recovery ${ }^{5-14}$ and spasticity reduction after stroke ${ }^{7,15}$. In this paper, a new EStim protocol - Breathing-controlled electrical stimulation (BreEStim), is introduced, according to recent research findings on the systemic effect of voluntary breathing ${ }^{16,17}$.

Transcutaneous electrical nerve stimulation (TENS) is a non-pharmacological modality for pain management ${ }^{1}$. TENS is noninvasive, inexpensive, safe and easy to use ${ }^{18}$. TENS is usually applied at varying frequencies, intensities, and pulse durations of stimuli for a prescribed treatment time. TENS has been applied to a variety of pain conditions, including neuropathic pain. The clinical effectiveness of TENS is controversial, particularly in spinal cord injury $\left(\mathrm{SCl}\right.$ ) and amputation (see Reviews ${ }^{1,19,20}$ ). The possible mechanisms are the gate control theory ${ }^{21}$ and the release of endogenous opioids 22,23 . Acupuncture from Traditional Chinese Medicine is another non-pharmacological modality for pain management. It has been well accepted in Western Medicine ${ }^{24}$. In modern acupuncture, the traditional acupuncture needle has been replaced by a surface electrode (or equivalent). A specialized electrode is place over traditional acupoints and an electrical stimulation is delivered. This modification has been termed electroacupuncture ${ }^{25,26}$. Needle acupuncture and electroacupuncture are both effective in analgesia via the release of endogenous opioids ${ }^{27,28}$. The effect of electroacupuncture is usually reliable, however the effect is dependent on intensity and frequency of delivered electrical stimulation. Different frequencies of electrical stimulation generate different endogenous opioids, and the analgesic effect is naloxone-reversible ${ }^{26,27}$. Recently, it has been found that repetitive painful stimulation (aversiveness) leads to significant pain attenuation. The induced pain attenuation is not naloxone-reversible ${ }^{29}$. Integrating of these pain coping mechanisms (acupuncture, electrical stimulation, aversiveness) into a possible new intervention could improve its clinical efficacy.

EMG-triggered neuromuscular stimulation has been used for many years to facilitate post-stroke motor recovery of finger extension impairments $5-10,12-14,34$. Recovery of hand function is important for stroke patients, and yet is very challenging. Roughly one third of all people who experience a stroke will have some residual impairment of the upper extremity ${ }^{30-32}$, with major impairments in hand function ${ }^{33}$. The EMGtriggered NMES intervention protocol involves initiation of a voluntary contraction of extensor muscles for a specific movement until the muscle activity reaches a threshold level. As soon as the EMG activity reaches a target threshold, an assisting electrical stimulus begins to facilitate the 
movements. This intervention protocol is superior to regular NMES in motor recovery ${ }^{6,7}$. Chae and $\mathrm{Yu}^{35}$ stated that all randomized controlled studies reported improvement in motor function using this intervention protocol, with mild to moderately impaired patients improving the most. It is most likely that this intervention takes advantage of active engagement of patients (by setting a target EMG threshold) and this results in measureable changes in recovery as well as documented changes in the cortex ${ }^{6,7}$. This is supported by a recent functional MRI study that showed a significant increase in cortical intensity in the ipsilateral somatosensory cortex after treatment in the NMES group, as compared with the control group ${ }^{36}$. Furthermore, electrical stimulation may also help to reduce spasticity after stroke, ${ }^{75}$, but the effect is short-lasting, about ${ }^{30}$ min after EStim ${ }^{37}$. In contrast, our recent invention of breathing-controlled electrical stimulation (BreEStim) has a long-lasting effect on spasticity reduction, even after a single session of treatment ${ }^{16}$.

Human breathing is a very unique motor act. It can be controlled reflexively (automatic breathing), e.g., during sleep, and also voluntarily when needed (voluntary breathing), e.g., singing, speech, etc. During voluntary breathing, humans need to voluntarily suppress autonomic control of breathing through voluntary cortical activation (the "cortical respiratory center") ${ }^{38,39}$. Brain imaging studies ${ }^{40-51}$ have demonstrated extensive respiratory-related involvement of cortical areas bilaterally, including the primary motor cortex (M1), the premotor cortex, the supplementary motor area, the primary and secondary somatosensory cortices, the insula, the anterior cingulate cortex and amygdala, and the dorsolatera prefrontal cortex. The insula is known to have strong connections to brainstem centers and is involved in pain processing ${ }^{52}$. During autonomic breathing, inspiration is active while expiration is passive, mainly relying on recoil force of the chest wall. Similarly, volitional inspiration activates more respiratory-related cortical and subcortical areas when compared to volitional expiration ${ }^{46}$. These cortical and subcortical areas activated during voluntary breathing are also involved in different functions ${ }^{53}$, such as muscle tone, pain, posture, mood, speech, etc. Therefore, it is not unreasonable to associate interactions in breathing with modulation of other functions.

Recently, we have discovered that there exist interactions between respiratory and motor systems during voluntary breathing. Specifically, there is a finger extension-inspiration coupling ${ }^{16,54-56}$. When electrical stimulation is delivered to the finger extensors during the inspiratory phase of voluntary breathing, a long-lasting effect of reduction in finger flexor spasticity (muscle tone) in chronic stroke patient is observed ${ }^{16}$. In another study ${ }^{17}$, shooting phantom pain in a patient with an above-the-knee amputation disappeared after the BreEStim treatment, but re-appeared ${ }^{28}$ days later after receiving a sustained electrical stimulation accidentally. This case study provides a unique opportunity to understand that the affective component of noxious stimuli of neuropathic pain (shooting phantom pain) has been modified by the BreEStim treatment, but then retriggered by an accidental stimulation. These observations of tone and pain reduction have demonstrated that voluntary breathing, inspiration in particular, could be integrated into an electrical stimulation paradigm to improve its efficacy in neuropathic pain management and post-stroke spasticity management.

\section{Case Presentations}

\section{Case 1: Post-stroke Spasticity Management}

The patient was a 69 years-old male who had right hemiplegia secondary to a stroke 22 months ago. He was medically stable and had been discharged from outpatient physical and occupational therapy programs. No brain imaging results were available at the time of experiments. $\mathrm{He}$ had weakness on his right side but was able to walk independently without an assistive device. He had residual voluntary finger flexion and extension, but with limited active range of motion at his right metacarpophalangeal (MCP) joints, from $90^{\circ}$ to $70^{\circ}$ of MCP flexion, i.e., not able to sufficiently open his hand and fingers for functional use. Muscle tone of his right finger flexors was moderately increased. Modified Ashworth Scale (MAS) was 1+. Sensation of his right hand and fingers, however, was intact to light touch. He received approximately a 30-min BreEStim to the finger extensors. His finger flexor spasticity decreased to minimum $(M A S=0)$ and voluntary finger extension became nearly normal immediately after the treatment. This patient regained his hand function as well. He reported that he could cut meat with a knife and button shirts using his impaired hand. More strikingly, the recovery retained at least 8 weeks during follow-up visits (Figure 1).

\section{Case 2: Neuropathic Pain Management}

The patient was a 40 years-old male who suffered a spinal cord injury 4.5 years ago in a motor vehicle accident, resulting in T8 ASIA A spinal cord injury. The patient complained of neuropathic pain at the injury level, while he had no other active medical issues. He had been stable on a pain regime for 2 weeks prior to the treatment. He received EStim (one session per day for five consecutive days) first, waited 1 week as a washout, and then received BreEStim with the same dose (one session per day for 5 consecutive days). Each treatment session consisted of 120 stimuli (EStim or BreEStim). Surface electrodes were placed on acupoints (Neiguan and Weiguan) of the right forearm. Modified Visual Analogue Scale (mVAS) was used to compare the effect of each intervention (EStim and BreEStim). As shown in Figure 2, BreEStim had a greater pain reduction effect than EStim, except for Day 2 during BreEStim when the patient had a urinary tract infection (UTI) which was successfully treated with antibiotics. The intensity of electrical stimulation was similar between EStim and BreEStim (Figure 2). He tolerated both interventions well (maximum output intensity from the stimulator was used), even during the UTI. During the entire experimental period (4 weeks), the subject maintained the same dose and schedule of pain medications. Both BreEStim and EStim treatment sessions were performed at the same time of the day (between 11 AM to Noon), such that changes in the pain rating could possibly be attributed to stimulation effects and not diurnal variation.

Protocol

The following BreEStim protocol could be applied for both finger flexor spasticity and neuropathic pain management. The main difference lies in surface electrode placement and adjustment of stimulation intensity. These differences are explained in detail for each application.

\section{Subject Preparation and Setup}

1. Seat the subject comfortably. Place the arms and hands comfortably on the treatment table.

2. Identify and localize the area of interest for surface electrode placement.

1. For spasticity management, palpate the muscle belly of finger extensors and confirm with electrical stimulation. 
2. For pain management, locate acupuncture points of Neiguan and Weiguan on the forearm ${ }^{24}$ ipsilateral to the side of interest, e.g., amputation $^{17}$, or on the side with more symptoms, e.g., SCl. Neiguan is located about 3 -finger width above the wrist crease on the volar side and in the middle between medial and lateral boards of the forearm (i.e., distal $1 / 6$ of the forearm $)^{24}$ (Figure 3). Weiguan is the counterpart of Neiguan, located in the dorsal aspect of the forearm ${ }^{24}$.

3. Trim each self-adhesive electrode to about a $2 \mathrm{~cm} \times 2 \mathrm{~cm}$ square to provide focal and isolated electrical stimulation.

1. For spasticity management, place the cathode over the finger extensor muscle belly (Figure 4). Attach the anode to a site $1 \sim 2 \mathrm{~cm}$ distal to the cathode. Optimize the sites for the anode and cathode when eliciting the largest and isolated finger extension response with a minimal wrist response.

2. For pain management, place the cathode electrode on Neiguan, and the anode electrode on Weiguan.

4. Connect surface electrodes to the electrical stimulator (Digitimer DS7A, UK, www.digitimer.com).

5. Place and secure facemask. Select the size of facemask carefully to fit individual's face to prevent air leakage and to provide comfort of wearing the mask (Figure 5).

6. Connect facemask to a pneumotach system (Series 1110A, Hans Rodolph Inc; Kansas City, Missouri; http://www.rudolphkc.com).

\section{Instruction on Voluntary Breathing}

Voluntary breathing, particularly voluntary inhalation, plays a critical role in this intervention. Voluntary inhalation is defined as effortful deep and fast inhalation. Instruct the subject to take a single isolated deep breath, similar to routine deep breaths, but faster and stronger. There is no need to perform voluntary exhalation preceding forced inhalation in a breathing cycle. Allow the subject to have $8 \sim 10$ practice trials to understand the instructions.

\section{Electrical Stimulation Settings}

1. Set a single electrical stimulus as a single square-wave pulse with $0.1 \mathrm{~ms}$ duration. The intensity of electrical stimulation is different for different applications. Since a single electrical stimulus is delivered each time, there is no need to set frequency parameter.

2. For spasticity management, determine the intensity of electrical stimulation when 1) isolated finger extension responses are elicited with minimal involvement of wrist joint responses; 2) the highest level that the subject could tolerate. The absolute magnitude of stimulation intensity could be different for different subjects. Encourage the highest intensity that the subject could tolerate to achieve the best outcome.

3. For pain management, allow the subject to determine incremental changes of the stimulation intensity. The starting intensity is zero. The highest level is the maximal output of the stimulator or the level the subject could tolerate. However, explicitly instruct the subject that discomfort or painfulness, even "aversiveness" of electrical stimulation is part of treatment; therefore, encourage the subject to select the highest level that the subject could tolerate.

\section{Control of Electrical Stimulation}

1. Write a customized LabView (National Instrument, Austin, TX) program to control delivery of electrical stimulation in two ways: BreEStim and EStim.

2. Breathing-controlled electrical stimulation (BreEStim) (Figure 6):

1. Determine the peak airflow rate during voluntary inhalation, i.e., during the deepest and fast inhalation.

2. Determine the threshold which is $40 \%$ peak airflow rate. Of note, set the threshold higher than the airflow rate during normal breathing to encourage deeper and faster voluntary breathing.

3. Then set the trigger function. When the instantaneous airflow rate of an isolated voluntary inhalation reaches or beyond the threshold, the LabView program triggers and delivers a single electrical stimulus with preset duration and intensity ${ }^{16}$. Allow the subject to rest upon request.

3. Randomly-triggered electrical stimulation (EStim):

1. Allow the subject breathe normally without specific instructions on breathing.

2. The LabView program randomly delivers a single electrical stimulus with preset duration and intensity every 4 to 7 sec. Similarly, allow the subject to rest upon request.

\section{Dose of BreEStim}

It is recommended that each session of treatment has 100 to 120 BreEStim stimuli. It lasts approximately $30-40$ min.

\section{Recording and Monitoring}

1. Make sure there is no air leakage from the facemask, since voluntary inhalation plays an important role in this protocol.

2. Monitor signs of hypoxemia and hyperventilation when the subject wears facemask. Allow rest at the subject's request for this purpose.

3. Record any side effect, tolerance of voluntary breathing via a face mask, and any psycho-social effects.

4. For pain management, record any reduction of pain, i.e., visual analogue scores (VAS) ${ }^{57}$ and duration of the effect. Use the modified VAS (mVAS) to further quantify the effect of pain reduction, i.e., how much pain is reduced and how long it lasts (reduction $\times$ hours). Also record the average intensity for each session, since the intensity of electrical stimulation varies during each session of treatment. 
5. For spasticity management, record the Modified Ashworth Scale (MAS) value of the target muscle and other clinical measurements, including strength, sensation, and range of motion.

\section{Representative Results}

Breathing-controlled electrical stimulation (BreEStim) has demonstrated excellent clinical efficacy in management of neuropathic pain in spinal cord injury and post-stroke finger flexor spasticity. Spasticity reduction after BreEStim treatment depends on the severity of pretreatment conditions. As shown in Figure 1, the finger flexor spasticity was greatly reduced after BreEStim treatment. Finger flexor spasticity has been reduced from MAS $1+$ to minimum (MAS $=0$ ). The patient is able to open his hand and fingers for functional use. It is important to note that other patients may not have the same degree of spasticity reduction and functional improvement. In a patient with severe finger flexor spasticity $(M A S=3$ ) and without residual voluntary finger extension (as shown in Figure 4), finger flexor spasticity was reduced to MAS=1. This makes it easier for the patient to range her fingers, but does not restore functional use of her hand.

BreEStim also demonstrates better and longer pain reduction effects. Figure 2 shows that with similar intensity of electrical stimulation, BreEStim has better outcome than regular electrical stimulation. However, BreEStim did not affect pain scores on Day 2 when the patient had a urinary tract infection. This suggests that BreEStim has no effect on pain reduction when pain is confounded by infection.

Voluntary breathing plays a key role in BreEStim. It is important to choose a facemask that fits the patient (Figure 5) to prevent air leakage. The airflow rate is relatively low during normal breathing (1.6 liter/sec, Figure 6). Forceful voluntary inhalation could greatly increase the airflow rate (about 8 liter/sec). Placement of surface electrodes is also important. As described in detail and shown in Figure $\mathbf{3}$ and Figure $\mathbf{4}$, placement for pain and spasticity management is different. It is necessary to note that location of muscle belly of finger extensors may change as a result of atrophy, deformity after stroke.
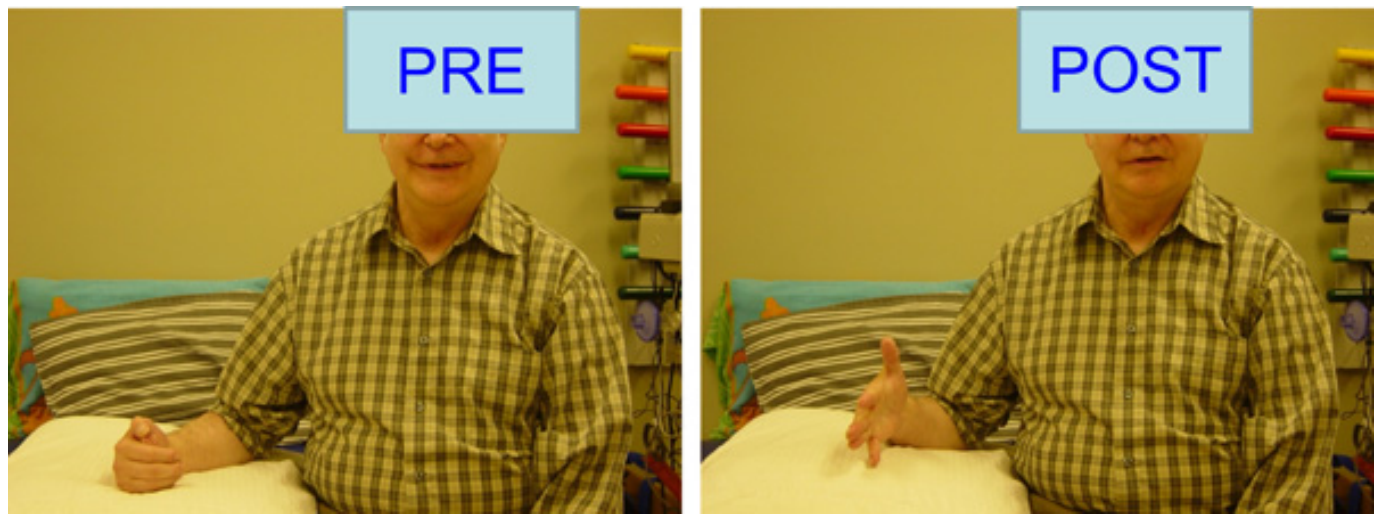

Figure 1. Comparison of hand posture pre- and post-BreEStim. The stroke patient could open his hand after BreEStim. 


\section{BreEStim}

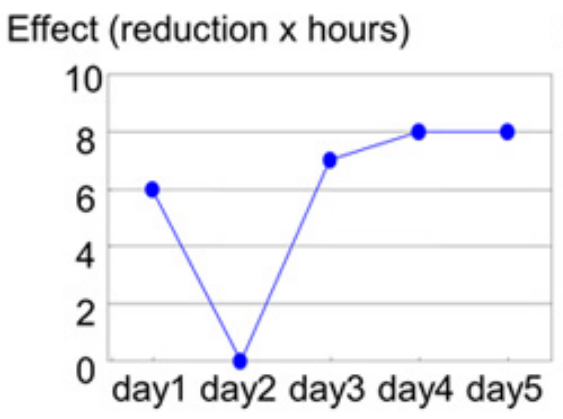

EStim

Effect (reduction $\mathrm{x}$ hours)

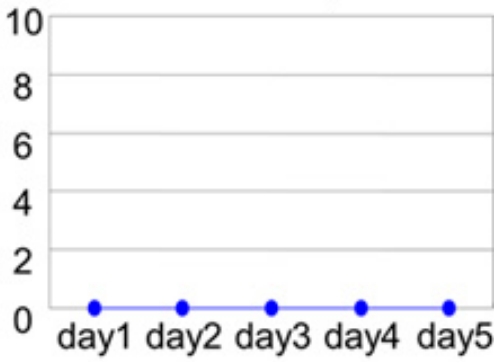

Stimulation intensity $(\mathrm{mA})$
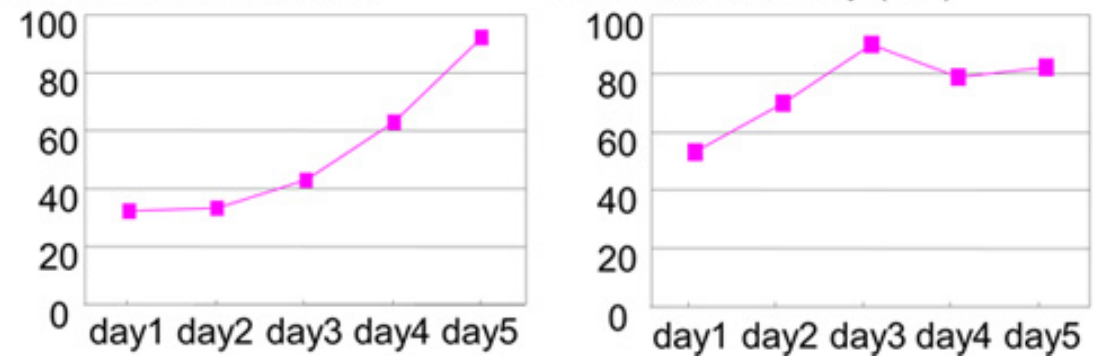

Figure 2. Comparison of BreEStim and EStim effects on pain reduction.

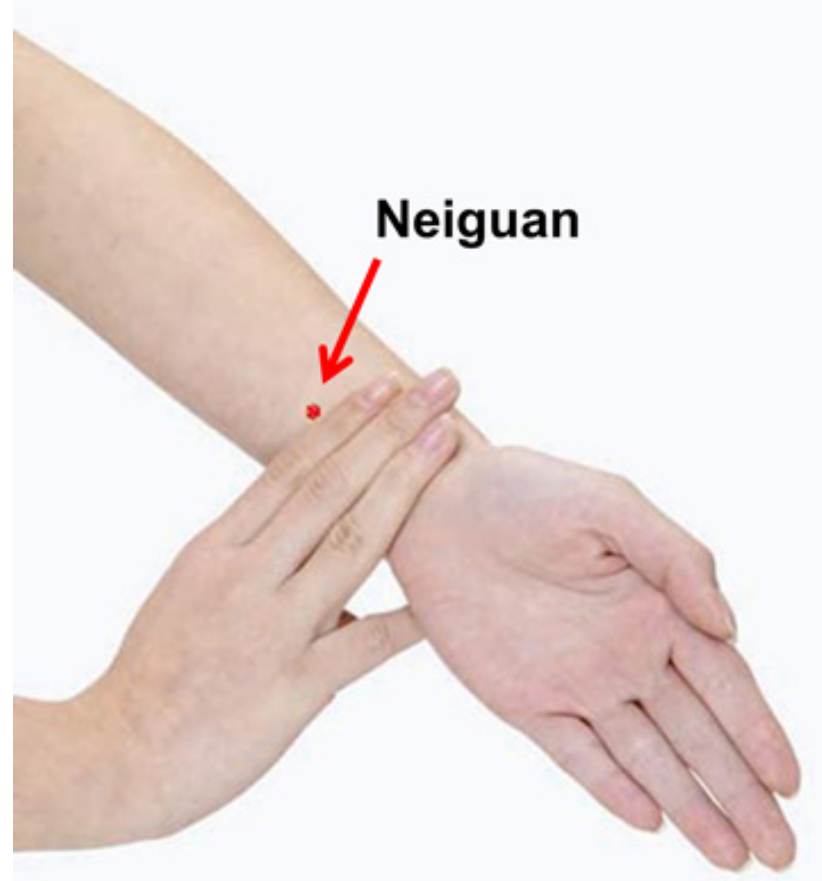

Figure 3. Location of Neiguan. Note, Waiguan is the counterpart of Neiguan on the dorsal aspect of the forearm. 


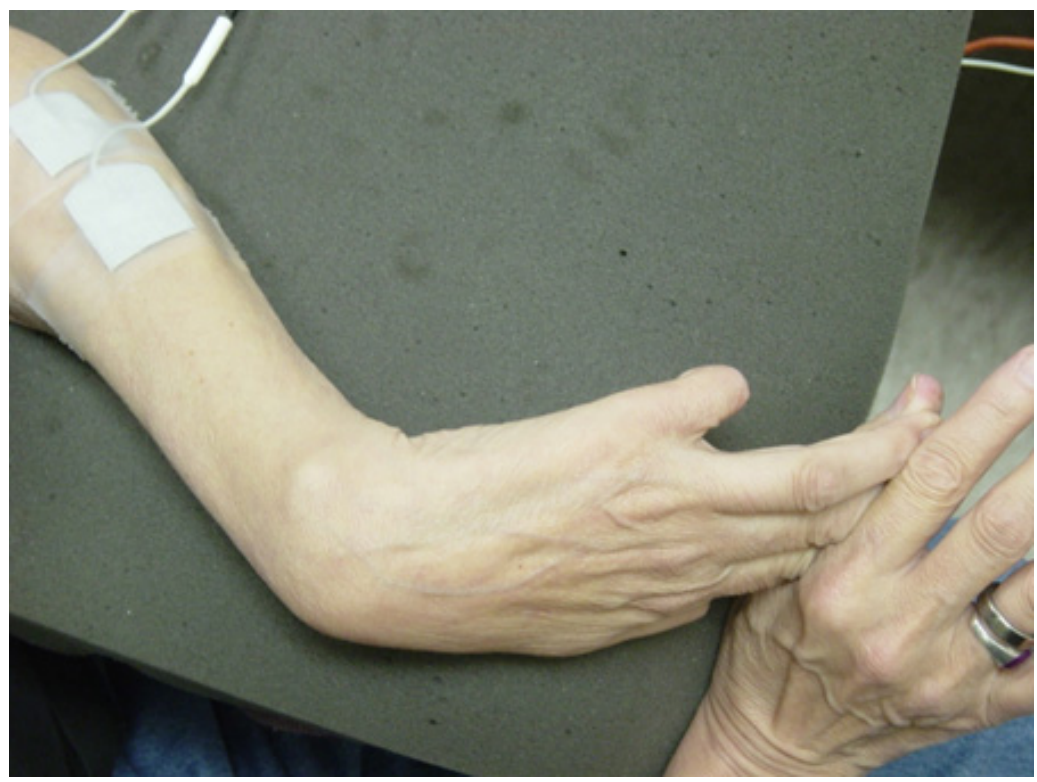

Figure 4. Placement of surface electrodes on finger extensors.

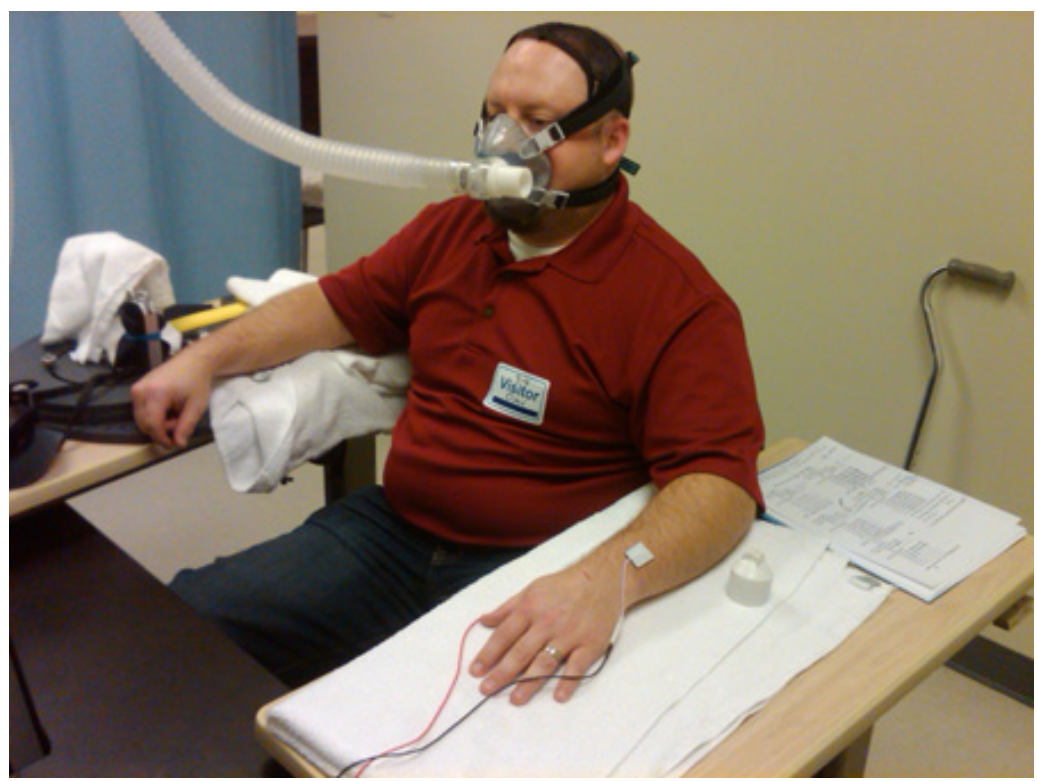

Figure 5. A patient during BreEStim. Surface electrodes are placed on Neiguan and Waiguan. 


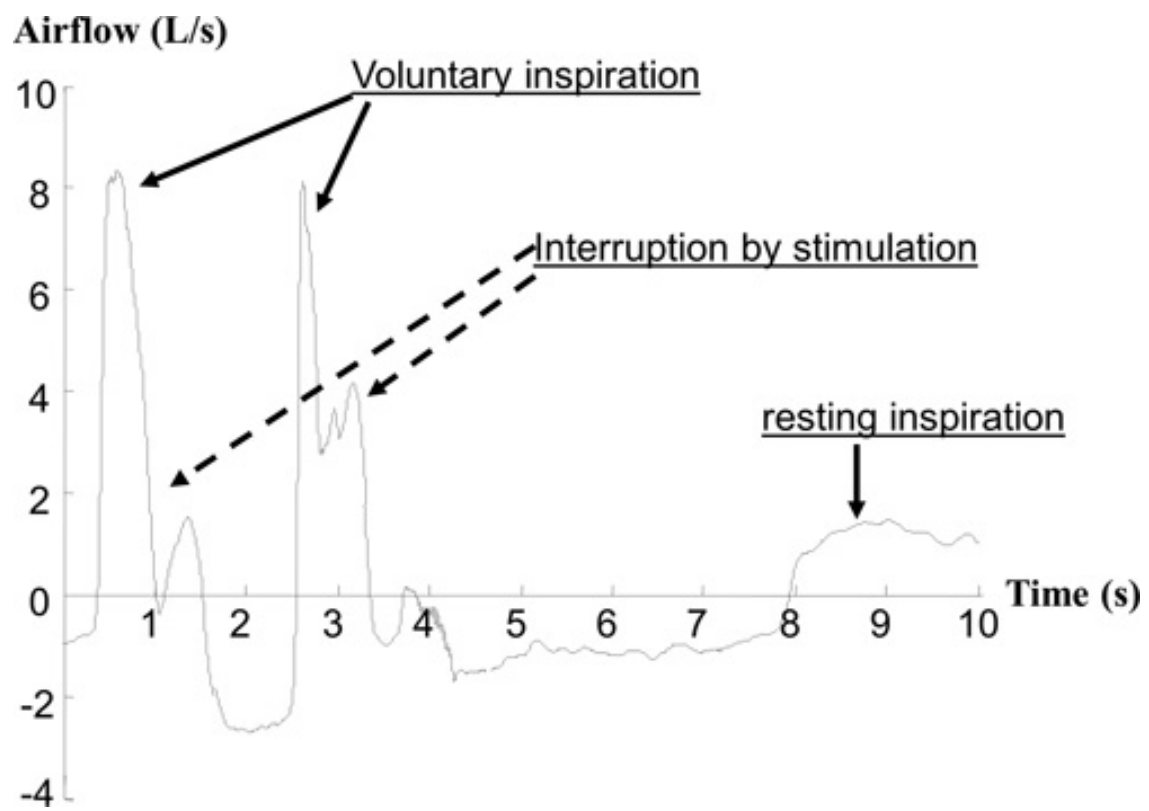

Figure 6. real-time measurement of airflow rate during voluntary inspiration and resting inspiration. Note that the airflow rate is much higher during voluntary inspiration than resting inspiration. It is noticeable that patient's breathing was interrupted by electrical stimulation.

\section{Discussion}

Breathing-controlled electrical stimulation (BreEStim), as shown in the above two cases, has demonstrated clinical efficacy in spasticity management and subsequent hand function recovery in chronic stroke patients ${ }^{16}$, as well as management of neuropathic pain of central origin in the above patient with a spinal cord injury or of peripheral origin in a patient with above-the-knee amputation ${ }^{17}$. This enhanced clinical outcome and broader clinical applications of BreEStim are attributed to its unique approach. Intervention with electrical stimulation targeted at the short window of voluntary breathing-associated cortical and subcortical activation ${ }^{40-51}$ could augment its clinical efficacy via intrinsic physiological coupling, e.g., respiratory-motor coupling for spasticity management ${ }^{16}$. In this intervention, voluntary breathing becomes critical, particularly voluntary inspiration. Education for patients on proper breathing techniques and accurate measurement of breathing parameters (e.g., no air leakage) are measures to prevent failure of the BreEStim intervention.

The new intervention protocol - BreEStim, has a few advantages, in addition to better efficacy and broader applications.

BreEStim is patient-centered. BreEStim encourages active engagement of patients since voluntary breathing is required ${ }^{17}$. Patients feel they actively participate in managing their pain, rather than "a passive participant in their own care". For example, the patient controls the intensity of electrical stimulation, starting from zero to the highest level that the patient could tolerate ${ }^{17}$. This may enhance their treatment compliance. EMGtriggered EStim also involves active participation ${ }^{36}$, but the patient can not control the intensity of electrical stimulation.

BreEStim takes an integrative, system-based approach. As demonstrated in an earlier study ${ }^{17}$, different pain coping mechanisms are integrated into one protocol, including electrical stimulation, acupuncture, aversive stimulation, and the systemic effects of voluntary breathing. As such, patients are able to tolerate high levels of electrical stimulation, leading to enhanced analgesic effects. Such a positive feedback loop (activation of the reward system) results in greater clinical efficacy. Using this integrative, system-based approach, certain signals of voluntary breathing could also be used to identify the time window of interactions between systems. As such, BreEStim could be applied to patients with severe spasticity. These patients are usually not able to perform voluntary contraction, thus "clean" EMG signals from the target muscle are not available. In EMG-triggered electrical stimulation, EMG signals from the targeted muscles (e.g., finger extensors) are required to trigger electrical stimulation. Therefore, application of EMG-triggered EStim is limited to patients with mild to moderate spasticity.

BreEStim is a non-invasive, non-pharmacological treatment. This is critical because patients often require a long-term use of medications, and most medications for chronic pain and spasticity have side effects that can sometimes be very severe. The possible side effects include addiction, overdose, withdrawal symptoms, and constipation, etc. These potential side effects could be avoided in the BreEStim treatment.

BreEStim is an alternative choice. The alternative non-pharmacological treatment with better analgesic effects is important, particularly when neuropathic pain is difficult to manage. For example, only $7 \%$ of responders reported pharmacological treatment is effective for neuropathic pain following $\mathrm{SCl}$ in a postal survey ${ }^{58}$.

In summary, this breathing-driven stimulation, BreEStim, is based on the newly discovered phenomenon of intrinsic physiological coupling activated during voluntary breathing. The BreEStim protocol has demonstrated clinical efficacy for neuropathic pain and post-stroke spasticity management. Further research is warranted to examine underlying mechanisms that mediate the intervention effect. Importantly, there may be other applications that have not yet discerned. 


\section{Disclosures}

Method and apparatus of breathing-controlled electrical simulation for skeletal muscles (inventor: S.L., pending patent, application number 12146176).

\section{Acknowledgements}

This study was supported in part by NIH grants (NIH/NINDS R01NS060774; NIH/NICHD/NCMRR R24 HD050821-08 under subcontract with Rehabilitation Institute of Chicago). The author thanks Craig Ditommaso, MD for his editing and helpful suggestions.

\section{References}

1. Sluka, K.A. \& Walsh, D. Transcutaneous electrical nerve stimulation: Basic science mechanisms and clinical effectiveness. J. Pain. 4, 109 (2003).

2. Sheffler, L.R., Hennessey, M.T., Naples, G.G., \& Chae, J. Peroneal nerve stimulation versus an ankle foot orthosis for correction of footdrop in stroke: impact on functional ambulation. Neurorehabil. Neural Repair. 20, 355-60 (2006).

3. Sheffler, L.R. \& Chae, J. Neuromuscular electrical stimulation in neurorehabilitation. Muscle Nerve. 35, 562 (2007).

4. Moe, J.H. \& Post, H.W. Functional electrical stimulation for ambulation in hemiplegia. J. Lancet. 82, 285-288 (1962)

5. de Kroon, J.R., ljzerman, M.J., Chae, J., Lankhorst, G.J., \& Zilvold, G. Relation between stimulation characteristics and clinical outcome in studies using electrical stimulation to improve motor control of the upper extremity in stroke. J. Rehabil. Med. 37, 65-74 (2005).

6. Bolton, D.A.E., Cauraugh, J.H., \& Hausenblas, H.A. Electromyogram-triggered neuromuscular stimulation and stroke motor recovery of arm/ hand functions: A meta-analysis. J. Neurologic. Sci. 223, 121 (2004).

7. Chae, J. Neuromuscular electrical stimulation for motor relearning in hemiparesis. Phys. Med. Rehabil. Clin. N. Am. 14, S93-109 (2003).

8. Chae, J., et al. Neuromuscular stimulation for upper extremity motor and functional recovery in acute hemiplegia. Stroke. 29, 975-9 (1998).

9. Cauraugh, J., Light, K., Kim, S., Thigpen, M., \& Behrman, A. Chronic motor dysfunction after stroke: recovering wrist and finger extension by electromyography-triggered neuromuscular stimulation. Stroke. 31, 1360-4 (2000).

10. Cauraugh, J.H. \& Kim, S. Two Coupled Motor Recovery Protocols Are Better Than One: Electromyogram-Triggered Neuromuscular Stimulation and Bilateral Movements. Stroke. 33, 1589-1594 (2002).

11. Cauraugh, J.H. \& Kim, S.B. Chronic stroke motor recovery: duration of active neuromuscular stimulation. J. Neurologic. Sci. 215, 13-19 (2003).

12. Crisan, R. \& Garner, C. Effectiveness of EMG-triggered muscular stimulation in outpatients with a stroke older than one year. Neurol. Rehabil. 7, 228 (2001).

13. Francisco, G., et al. Electromyogram-triggered neuromuscular stimulation for improving the arm function of acute stroke survivors: a randomized pilot study. Arch. Phys. Med. Rehabil. 79, 570-5 (1998).

14. Heckmann, J., et al. EMG-triggered electrical muscle stimulation in the treatment of central hemiparesis after a stroke. Euro. J. Phys. Med. Rehabil. 7, 138 (1997).

15. Bakhtiary, A.H. \& Fatemy, E. Does electrical stimulation reduce spasticity after stroke? A randomized controlled study. Clin. Rehabil. 22, 418-25 (2008).

16. Li, S. \& Rymer, W.Z. Voluntary breathing influences corticospinal excitability of nonrespiratory finger muscles. J. Neurophysiol. 105, 512-21 (2011).

17. Li, S., Melton, D.H., \& Berliner, J.C. Breathing-controlled electrical stimulation (BreEStim) could modify the affective component of neuropathic pain after amputation: a case report. J. Pain Res. 5, 71-5 (2012).

18. Norrbrink Budh, C. \& Lundeberg, T. Non-pharmacological pain-relieving therapies in individuals with spinal cord injury: a patient perspective. Complement Ther. Med. 12, 189-97 (2004).

19. Mulvey, M.R., Bagnall, A.M., Johnson, M.I., \& Marchant, P.R. Transcutaneous electrical nerve stimulation (TENS) for phantom pain and stump pain following amputation in adults. Cochrane Database Syst. Rev., CD007264 (2010).

20. Johnson, M.I. \& Bjordal, J.M. Transcutaneous electrical nerve stimulation for the management of painful conditions: Focus on neuropathic pain. Expert Rev. Neurotherap. 11, 735 (2011).

21. Melzack, R. \& Wall, P.D. Pain mechanisms: a new theory. Science. 150, 971-9 (1965).

22. Kalra, A., Urban, M.O., \& Sluka, K.A. Blockade of opioid receptors in rostral ventral medulla prevents antihyperalgesia produced by transcutaneous electrical nerve stimulation (TENS). J. Pharmacol. Exp. Ther. 298, 257-63 (2001).

23. Sluka, K.A., Deacon, M., Stibal, A., Strissel, S., \& Terpstra, A. Spinal blockade of opioid receptors prevents the analgesia produced by TENS in arthritic rats. J. Pharmacol. Exp. Ther. 289 (2), 840-6 (1999).

24. Deadman, P., Al-khafaji, M., \& Baker, K. A manual of acupuncture. Eastland Press, Visa, California, (2007).

25. Han, J.S., Li, S.J., \& Tang, J. Tolerance to electroacupuncture and its cross tolerance to morphine. Neuropharmacol. 20, 593-6 (1981).

26. Han, J.S. Acupuncture and endorphins. Neurosci. Lett. 361, 258 (2004).

27. Huang, C., Wang, Y., Han, J.S., \& Wan, Y. Characteristics of electroacupuncture-induced analgesia in mice: variation with strain, frequency, intensity and opioid involvement. Brain Res. 945, 20-5 (2002).

28. Wan, Y., Wilson, S.G., Han, J., \& Mogil, J.S. The effect of genotype on sensitivity to electroacupuncture analgesia. Pain. 91, 5-13 (2001).

29. Rennefeld, C., Wiech, K., Schoell, E.D., Lorenz, J., \& Bingel, U. Habituation to pain: Further support for a central component. Pain. 148, 503 (2010).

30. Gray, C.S., et al. Motor recovery following acute stroke. Age Ageing. 19, 179-84 (1990).

31. Nakayama, H., Jorgensen, H.S., Raaschou, H.O., \& Olsen, T.S. Recovery of upper extremity function in stroke patients: the Copenhagen Stroke Study. Arch. Phys. Med. Rehabil. 75, 394-8 (1994).

32. Parker, V.M., Wade, D.T., \& Langton Hewer, R. Loss of arm function after stroke: measurement, frequency, and recovery. Int. Rehabil. Med. 8, 69-73 (1986). 
33. Trombly, C.A. In: Occupational therapy for physical dysfunction., Trombly, C.A., ed., Williams \& Wilkins, Baltimore, $454-471$ (1989).

34. Bocker, B. \& Smolenski, U.C. Training by EMG-triggered electrical muscle stimulation in hemiparesis. 13, 139 (2003).

35. Chae, J. \& Yu, D. A critical review of neuromuscular electrical stimulation for treatment of motor dysfunction in hemiplegia. Assist Technol. 12, 33-49 (2000).

36. Kimberley, T.J., et al. Electrical stimulation driving functional improvements and cortical changes in subjects with stroke. Exp. Brain Res. 154, 450-60 (2004).

37. Dewald, J.P., Given, J.D., \& Rymer, W.Z. Long-lasting reductions of spasticity induced by skin electrical stimulation. IEEE Trans. Rehabil. Eng. 4, 231-42 (1996).

38. Haouzi, P., Chenuel, B., \& Barroche, G. Interactions between volitional and automatic breathing during respiratory apraxia. Respir Physiol. Neurobiol. 152, 169-75 (2006).

39. Guz, A. Brain, breathing and breathlessness. Respir. Physiol. 109, 197-204 (1997).

40. Colebatch, J.G., et al. Regional cerebral blood flow during volitional breathing in man. J. Physiol. 443, 91-103 (1991).

41. Maskill, D., Murphy, K., Mier, A., Owen, M., \& Guz, A. Motor cortical representation of the diaphragm in man. J. Physiol. 443, 105-21 (1991).

42. Ramsay, S.C., et al. Regional cerebral blood flow during volitional expiration in man: a comparison with volitional inspiration. J. Physiol. 461, 85-101 (1993).

43. Fink, G.R., et al. Hyperpnoea during and immediately after exercise in man: evidence of motor cortical involvement. J. Physiol. 489 (Pt 3), 663-75 (1995).

44. Macey, K.E., et al. fMRI signal changes in response to forced expiratory loading in congenital central hypoventilation syndrome. J. Appl. Physiol. 97, 1897-907 (2004).

45. Macey, P.M., et al. Functional magnetic resonance imaging responses to expiratory loading in obstructive sleep apnea. Respir. Physiol. Neurobiol. 138, 275-90 (2003).

46. Evans, K.C., Shea, S.A., \& Saykin, A.J. Functional MRI localisation of central nervous system regions associated with volitional inspiration in humans. J Physiol. 520 Pt 2, 383-92 (1999).

47. Smejkal, V., Druga, R., \& Tintera, J. Control of breathing and brain activation in human subjects seen by functional magnetic resonance imaging. Physiol Res. 48, 21-5 (1999).

48. Smejkal, V., Druga, R., \& Tintera, J. Brain activation during volitional control of breathing. Physiol Res. 49, 659-63 (2000).

49. Mazzone, S.B., McLennan, L., McGovern, A.E., Egan, G.F., \& Farrell, M.J. Representation of Capsaicin-evoked Urge-to-Cough in the Human Brain Using Functional Magnetic Resonance Imaging. Am. J. Respir. Crit. Care Med. 176, 327-332 (2007).

50. Evans, K.C. Cortico-limbic circuitry and the airways: Insights from functional neuroimaging of respiratory afferents and efferents. Biol. Psychol. 84, 13 (2010).

51. Evans, K.C., et al. Modulation of spontaneous breathing via limbic/paralimbic-bulbar circuitry: An event-related fMRI study. Neurolmage. 47, 961 (2009).

52. Tsumori, T., et al. Insular cortical and amygdaloid fibers are in contact with posterolateral hypothalamic neurons projecting to the nucleus of the solitary tract in the rat. Brain Res. 1070, 139-44 (2006).

53. Subramanian, H.H., Balnave, R.J., \& Holstege, G. The midbrain periaqueductal gray control of respiration. J. Neurosci. 28, 12274 (2008).

54. Li, S. \& Laskin, J.J. Influences of ventilation on maximal isometric force of the finger flexors. Muscle Nerve. 34, 651-655 (2006).

55. Li, S. \& Yasuda, N. Forced ventilation increases variability of isometric finger forces. Neurosci. Lett. 412, $243-247$ (2007).

56. Ikeda, E.R., et al. The valsalva maneuver revisited: the influence of voluntary breathing on isometric muscle strength. J. Strength Cond. Res. 23, 127-32 (2009).

57. McCarthy, M., Jr., et al. Visual analog scales for assessing surgical pain. J. Am. Coll. Surg. 201, 245-52 (2005).

58. Finnerup, N.B., Johannesen, I.L., Sindrup, S.H., Bach, F.W., \& Jensen, T.S. Pain and dysesthesia in patients with spinal cord injury: A postal survey. Spinal Cord. 39, 256-62 (2001). 